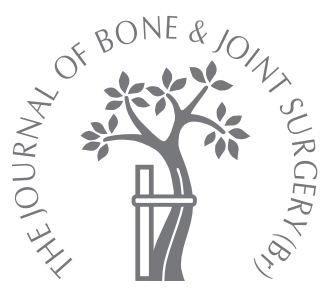

\title{
Subtalar and talonavicular arthrodesis through a single medial approach for the correction of severe planovalgus deformity
}

\author{
M. Knupp, \\ R. Schuh, \\ S. A. S. Stufkens, \\ L. Bolliger, \\ B. Hintermann \\ From Kantonsspital \\ Liestal, Liestal, \\ Switzerland
}

\begin{abstract}
We describe a retrospective review of the clinical and radiological parameters of $\mathbf{3 2}$ feet in 30 patients ( 10 men and 20 women) who underwent correction for malalignment of the hindfoot with a modified double arthrodesis through a medial approach. The mean followup was 21 months (13 to 37). Fusion was achieved in all feet at a mean of 13 weeks (6 to 30 ). Apart from the calcaneal pitch angle, all angular measurements improved significantly after surgery. Primary wound healing occurred without complications.

The isolated medial approach to the subtalar and talonavicular joints allows good visualisation which facilitated the reduction and positioning of the joints. It was also associated with fewer problems with wound healing than the standard lateral approach.
\end{abstract}

Triple arthrodesis of the talocalcaneal, talonavicular, and calcaneocuboid joints has been used to treat the sequelae of paralytic disease. ${ }^{1-3}$ The aim is to achieve a plantigrade foot stable in the frontal plane. It has further been employed to restore a painful and deformed hindfoot. Good outcomes have been reported. ${ }^{4-10}$ However, various complications, including problems with wound healing and superficial infections on the lateral side, have been described. ${ }^{4,5,7-9,11}$ In the long term, a high prevalence of subsequent arthritis of the adjacent joints ${ }^{4,5,7-9}$ and loss of correction ${ }^{4,5,12}$ have been noted. The traditional technique has involved two incisions in order to fuse the three joints. A single medial approach, preserving the calcaneocuboidal joint, may lead to comparably good results and may diminish the risk of degeneration in the neighbouring joints. ${ }^{13,14}$ It can reduce the complications of a lateral wound, particularly in high-risk patients. ${ }^{14-16}$ The object of this study was to assess radiologically the extent to which isolated subtalar and talonavicular joint fusion can correct deformity of the hindfoot, and to review the early complications after surgery.

\section{Patients and Methods}

Between July 2005 and July 2007, 30 patients (10 men and 20 women; 32 feet) with a large fixed valgus deformity underwent correction by fusion of the subtalar and talonavicular joints through a single medial approach. Their mean age was 71 years (52 to 86 ). The mean follow-up was for 21 months (13 to 37). Four patients also required medial displacement calcaneal osteotomy. Treatment of five patients with rheumatoid arthritis with methotrexate continued during the peri-operative period, with oral steroid medication limited to $10 \mathrm{mg}$ where possible; four patients had diabetes mellitus. All patients were evaluated clinically and radiologically according to standard protocols of our department, which include weightbearing anteroposterior and lateral radiographs of the entire foot. Patients with a previous arthrodesis of the hindfoot or with followup of less than 12 months were excluded from the study.

Technique of operation. A modification of the approach described by De Wachter et $\mathrm{al}^{13}$ and Jeng, Tankson and Myerson ${ }^{17}$ was used. A $6 \mathrm{~cm}$ long skin incision was made from the navicular towards the medial malleolus, parallel to and approximately $5 \mathrm{~mm}$ above the tendon of tibialis posterior. The tendon sheath was opened and the tendon inspected. In 12 cases with marked tendinosis the tendon was excised. The capsule of the talonavicular was then incised. A spreader was placed dorsolaterally to open the joint, which was subsequently denuded with a chisel. Next, the calcaneal wall was exposed down to the sustentaculum. A K-wire was inserted into the base of the sustentaculum and then used to place the spreader over the talocalcaneal joint. Taking care not to damage the anterior fibres of the deltoid ligament, the subtalar joint was opened and denuded with a chisel and a curette, and the exposed articular surfaces 


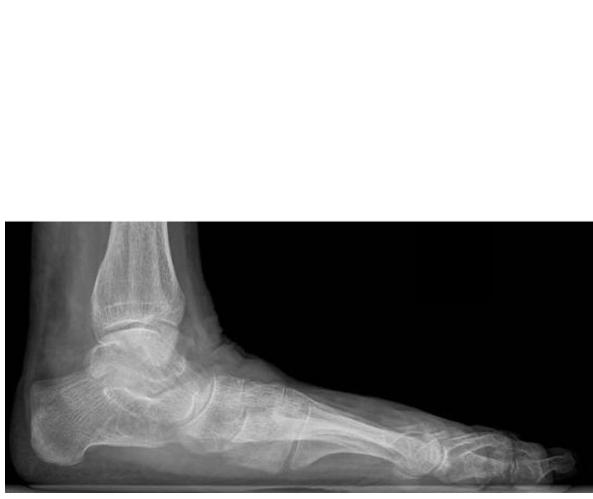

Fig. 1

Pre-operative radiographs of a 63 -year-old woman with symptomatic flatfoot.

feathered or drilled with a $2.0 \mathrm{~mm}$ drill bit. Bone grafts taken from the ipsilateral iliac crest were inserted in five cases. The foot was then held in a neutral position and Kwires were used to secure the correction. If the correction of the valgus deformity was considered insufficient, a medial displacement osteotomy of the calcaneum was added before the subtalar joint was fixed. This was needed in four cases. For the osteotomy an additional lateral approach was chosen. The lateral incision was slightly curved and about $1 \mathrm{~cm}$ posterior and parallel to the peroneal tendons. A small Hohmann retractor was inserted on the plantar aspect of the calcaneum and another over the calcaneum in the dorsal concavity. An oscillating saw was used to create the osteotomy and an osteotome to finalise the cut. A large lamina spreader was then used to mobilise the fragment. Finally, a Hohmann retractor was used to slide the calcaneal tuberosity medially for about $1 \mathrm{~cm}$. The overhanging bone was tapped down with a pin punch and hammer. Fixation was achieved by cannulated compression screws. The subcutaneous tissues and the skin were closed with interrupted sutures.

Three cannulated $4.0 \mathrm{~mm}$ compression screws for the talonavicular joint and two cannulated $6.5 \mathrm{~mm}$ compression screws (Newdeal-Integra Life Sciences, Plainsboro, New Jersey) for the subtalar joint were used to achieve a stable fixation (Figs 1 and 2). In cases where a calcaneal osteotomy was performed, a second posterior screw was added to secure the tuberosity. Lengthening of the tendo Achillis was performed in three cases. Lengthening of the peroneal tendons ${ }^{14,15}$ was not required.

Plain radiographs. Weight-bearing anteroposterior (AP) and lateral radiographs of the entire foot were taken and evaluated with a high-resolution monitor using the DICOM/PACS (TBS Computer-Systeme GMbH, Waakirchen, Germany) review application E-Film (Le Neg SA, Chexbres, Switzerland).

Correction of the deformity in the AP and lateral weight-bearing positions was measured by comparing pre-operative radiographs with those taken at final

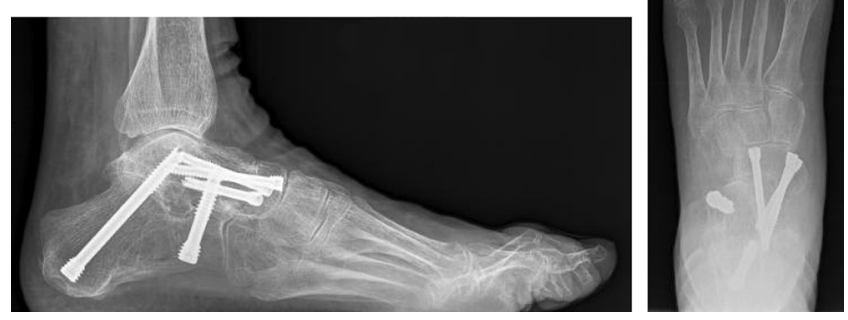

Fig. 2

Post-operative radiographs of the same patient as in Figure 1.

follow-up. The AP talonavicular coverage angle ${ }^{18}$ the AP talus-first metatarsal angle, ${ }^{18}$ the lateral talus-first metatarsal angle ${ }^{18}$ the lateral talocalcaneal angle ${ }^{18}$ and the calcaneal pitch angle ${ }^{19}$ were measured. The presence or absence of fusion of the talocalcaneal and talonavicular joints was recorded. The radiographs were evaluated by a board-certified orthopaedic surgeon $(\mathrm{BH})$ working in the foot and ankle unit who was not directly involved in the clinical aspects of the study.

Clinical assessment. The wounds were assessed during the in-patient stay and two, six and 12 weeks after surgery. Evidence of infection or breakdown of the wound was noted. Finally, the patients were asked to rate the success of the procedure (satisfied/dissatisfied).

Statistical analysis. Data were analysed using SPSS version 9.0 (SPSS Inc., Chicago, Illinois) and SigmaPlot 2004 for Windows (Systat Software Inc., Chicago, Illinois). Experimental data are presented as mean (SD), with minimum and maximum values and $95 \%$ confidence intervals (CI). In order to determine whether the data conform to Gaussian normal distribution, we performed the Kolmogorov-Smirnov normality test. Unpaired two-tailed Student's $t$-tests were used to analyse differences between the pre- and post-operative measures. A $\mathrm{p}$-value $<0.05$ was considered to be statistically significant.

\section{Results}

The radiological parameters and analyses are summarised in Table I. The mean AP talonavicular coverage angle improved significantly from $32^{\circ}$ (SD 18) (95\% CI 25 to 38) pre-operatively to $12^{\circ}$ (SD 12) (95\% CI 7 to 16) postoperatively $(\mathrm{p}<0.001)$. The mean AP talus-first metatarsal angle improved significantly from $16^{\circ}$ (SD 11) $(95 \%$ CI 12 to $21^{\circ}$ (pre-operatively to $7^{\circ}$ (SD 11) $(95 \%$ CI 3 to $12)$ post-operatively $(\mathrm{p}<0.001)$. The mean lateral talusfirst metatarsal angle increased significantly from $-19^{\circ}$ (SD 12) $(95 \%$ CI -23 to -14$)$ pre-operatively to $-8^{\circ}$ (SD 8$)(95 \%$ CI -11 to -5$)$ post-operatively $(\mathrm{p}<0.001)$. There was a significant improvement $(\mathrm{p}<0.001)$ in the mean lateral talocalcaneal angle from $41^{\circ}$ (SD 9) $(95 \%$ CI 38 to 42) pre- 


\begin{tabular}{|c|c|c|c|}
\hline & Pre-operative & Post-operative & p-value \\
\hline \multicolumn{4}{|c|}{ Anteroposterior talonavicular coverage angle $\left({ }^{\circ}\right)$} \\
\hline Mean (SD) & $32(18)$ & $12(12)$ & \\
\hline $95 \% \mathrm{Cl}^{*}$ & 25 to 38 & 7 to 16 & $<0.001$ \\
\hline Range & -21 to 83 & -22 to 38 & \\
\hline \multicolumn{4}{|c|}{ Anteroposterior talus-first metatarsal angle $\left({ }^{\circ}\right)$} \\
\hline Mean (SD) & $16(11)$ & $7(11)$ & \\
\hline $95 \% \mathrm{Cl}$ & 12 to 21 & 3 to 12 & $<0.001$ \\
\hline Range & -19 to 42 & -17 to 27 & \\
\hline \multicolumn{4}{|c|}{ Lateral talus 1 st metatarsal angle $\left({ }^{\circ}\right)$} \\
\hline Mean (SD) & $-19(12)$ & $-8(8)$ & \\
\hline $95 \% \mathrm{Cl}$ & -23 to -14 & -11 to 5 & $<0.001$ \\
\hline Range & -50 to 3 & -29 to 2 & \\
\hline \multicolumn{4}{|c|}{ Lateral talocalcaneal angle $\left({ }^{\circ}\right)$} \\
\hline Mean (SD) & $41(9)$ & $30(6)$ & \\
\hline $95 \% \mathrm{Cl}$ & 38 to 42 & 28 to 32 & $<0.001$ \\
\hline Range & 28 to 62 & 18 to 40 & \\
\hline \multicolumn{4}{|c|}{ Calcaneal pitch angle $\left({ }^{\circ}\right)$} \\
\hline Mean (SD) & $18(6)$ & $18(5)$ & \\
\hline $95 \% \mathrm{Cl}$ & 15 to 20 & 16 to 20 & $\begin{array}{l}0.360- \\
\mathrm{NS}^{\dagger}\end{array}$ \\
\hline Range & 2 to 33 & 6 to 32 & \\
\hline
\end{tabular}

* $95 \% \mathrm{Cl}, 95 \%$ confidence interval

† NS, not significant

operatively to $30^{\circ}$ (SD 6) $(95 \%$ CI 28 to 32$)$ postoperatively. A mean calcaneal pitch angle of $18^{\circ}$ (SD 6) (95\% CI 15 to 20 ) was observed pre-operatively and $18^{\circ}$ (SD 5) $(95 \%$ CI 16 to 20$)$ post-operatively ( $\mathrm{p}=0.360)$.

All patients showed radiological evidence of union in the fused joints, as illustrated in the post-operative radiographs of the patient shown in Figures 1 and 2. No patient had a symptomatic calcaneocuboidal joint or a malunion. The mean time to definitive radiological fusion was 13 weeks (6 to 30 ). The overall clinical results were graded as excellent in 16 patients $(53 \%)$, good in nine $(30 \%)$, fair in four $(13 \%)$ and poor in one $(3 \%)$.

Complications. Complications were limited to problems with superficial wound healing in three patients, who were subsequently treated with antibiotics for seven days. One patient with diabetes developed a cast-related pressure ulcer. The others, who did not have pre-existing rheumatoid arthritis or diabetes mellitus, developed a superficial infection after a haematoma. However, no deep infections, dehiscence or breakdowns of the wound were noted. Removal of the screws was carried out in four cases, and breakage of a screw in the talonavicular fusion was noted in one case. There was no loss of correction in any of the patients.

\section{Discussion}

In this retrospective review, isolated talonavicular and subtalar joint fusion using a single medial approach for correction of hindfoot deformity resulted in good radiological outcomes and few early complications. Significant improvement was observed in all angular measurements, with the exception of the calcaneal pitch angle. Union was achieved in all feet at a mean of 13 weeks after surgery, and early complications were limited to problems with superficial wound healing.

Historically, triple arthrodesis has been used to treat the sequelae of paralytic disease, with the aim of establishing a stable, plantigrade foot. ${ }^{1-3}$ The technique was later extended to correct painful deformities and arthritic joints. Triple arthrodesis traditionally involves two incisions and the technique has undergone only limited modification since it was first described. ${ }^{4,7,11}$ Recent studies have suggested that a double arthrodesis in non-paralytic feet can achieve comparably good results while preserving the calcaneocuboidal joint. ${ }^{13,15,20}$

The traditional two-incision technique has been shown to increase the risk of a residual supination deformity in patients with severe peritalar subluxation. ${ }^{21}$ The single medial incision allows for improved visualisation and exposure of the transverse tarsal joints. ${ }^{11,12}$ Malunion was not seen in any of our patients. We have found that this approach helps to mobilise and reduce the joints. The improved visualisation facilitates debridement of the joints without placing the posteromedial structures, especially the tendon of flexor hallucis longus, at risk. Placing the incision directly over the apex of the deformity allows good control of the position of the fused joints. 


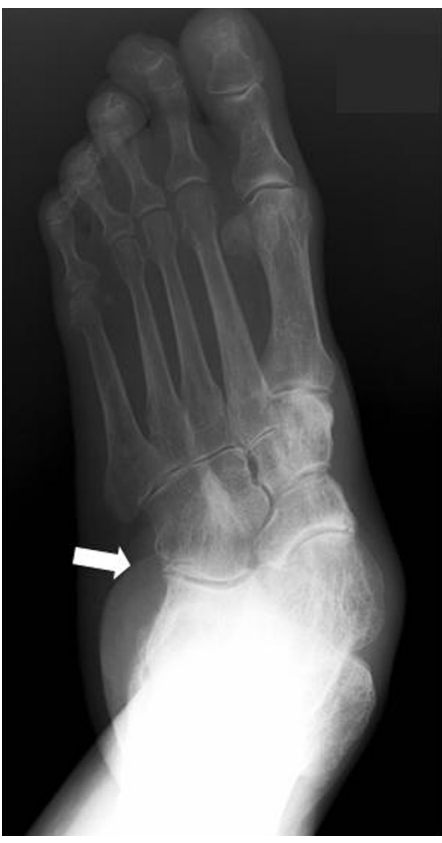

Fig. 3a

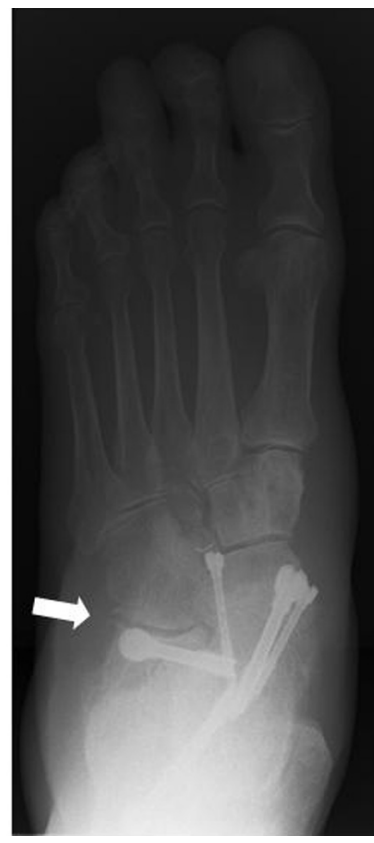

Fig. 3b

a) Pre- and b) intra-operative radiographs showing opening of the calcaneocuboid joint (arrow).

Previous studies of transverse tarsal and subtalar fusion in patients with arthritic changes or hindfoot deformity have described high rates of patient satisfaction, with improvements in function. However, a variety of complications have been encountered, particularly in patients with severe flatfoot deformity. ${ }^{4,5,7-9,11}$ Breakdown of the lateral wound has also been a problem. ${ }^{4,5,7-9,11}$ This may arise if correction of the deformity leads to increased tension on the lateral side of the foot. A single medial incision will reduce the risk of problems with wound healing, particularly in patients with severe deformity or deficient lateral skin. ${ }^{13-16}$

A cadaver study showed that much of the deformity may be corrected once the talonavicular joint is adequately reduced. ${ }^{22}$ The authors concluded that isolated talonavicular fusion is as effective as triple arthrodesis or talonavicular and calcaneocuboid fusion in correcting all aspects of flatfoot deformity. ${ }^{22}$ In a severe deformity, however, residual heel valgus is often present even after adequate reduction of the talonavicular joint. The remaining valgus deformity of the hindfoot can be addressed by subtalar fusion, which may be augmented by a medial displacement osteotomy of the calcaneum.

Sparing the calcaneocuboid joint has several advantages. It reduces operative time and the risk of nonunion. ${ }^{4,20}$ Reduction of the abducted foot is easier if the lateral column is not further shortened by fusing the calcaneocuboid joint. Retention of the calcaneocuboid joint provides some movement ${ }^{23,24}$ and thereby diminishes the loading on adjacent joints which may lead to arthritis., ${ }^{4,5,7-9}$ Our study showed that the modified double arthrodesis resulted in good correction of

nearly all parameters. We also observed distraction of the calcaneocuboid joint (Fig. 3), which may reduce the risk of degenerative joint disease at this site. Clinical assessments indicated that none of the patients experienced any symptoms in the calcaneocuboid joint after surgery.

Modification of the classic triple arthrodesis to a subtalar and talonavicular double arthrodesis using a single medial approach provides effective correction of hindfoot deformity in non-paralysed patients. The single medial exposure allows good visualisation of the joints, thereby facilitating reduction and reliable control of the positioning during surgery, and complications with wound healing were infrequent.

We wish to thank S Pannhorst, Kantonsspital Liestal, Switzerland, for her invaluable assistance with the statistical analyses in this study.

No benefits in any form have been received or will be received from a commercial party related directly or indirectly to the subject of this article.

\section{References}

1. Lambrinudi C. New operation on drop-foot. Br J Surg 1927;15:193-200.

2. Ryerson EW. Arthrodesing operations on the feet. J Bone Joint Surg 1923:5:453-71.

3. Wetmore RS, Drennan JC. Long-term results of triple arthrodesis in Charcot-MarieTooth disease. J Bone Joint Surg [Am] 1989;71-A:417-22.

4. Graves SC, Mann RA, Graves KO. Triple arthrodesis in older adults: results after longterm follow-up. J Bone Joint Surg [Am] 1993;75-A:355-62.

5. Figgie MP, O'Malley MJ, Ranawat C, Inglis AE, Sculco TP. Triple arthrodesis in rheumatoid arthritis. Clin Orthop 1993;292:250-4.

6. Angus PD, Cowell HR. Triple arthrodesis: a critical long-term review. J Bone Joint Surg [Br] 1986;68-B:260-5.

7. Saltzman CL, Fehrle MJ, Copper RR, Spencer E, Ponseti IV. Triple arthrodesis: twenty-five and forty-four-year average follow-up of the same patients. J Bone Joint Surg [Am] 1999:81-A:1391-402.

8. Pell RF, Myerson MS, Schon LC. Clinical outcome after primary triple arthrodesis. J Bone Joint Surg [Am]2000;82-A:47-57.

9. Knupp M, Skoog A, Törnkvist H, Ponzer S. Triple arthrodesis in rheumatoid arthritis: a retrospective long-term study of 32 cases. Foot Ankle Int 2008;29:297-7.

10. Trieb K. Management of the foot in rheumatoid arthritis. J Bone Joint Surg [Br]2005;87B:1171-7.

11. Rosenfeld PF, Budgen SA, Saxby TS. Triple arthrodesis is bone grafting necessary?: the results in 100 consecutive cases. J Bone Joint Surg [Br]2005;87-B:175-8.

12. Maenpaa $\mathbf{H}$, Lehto MU, Belt EA. What went wrong in triple arthrodesis?: an analysis of failure in 21 patients. Clin Orthop 2001;391:218-23.

13. De Wachter J, Knupp M, Hintermann B. Double-hindfoot arthrodesis through a single medial approach. Techniques Foot Ankle Surgery 2007;6:1-6.

14. Jackson WFM, Tryfonidis M, Cooke PH, Sharp RJ. Arthrodesis of the hindfoot for valgus deformity: an entirely medial approach. J Bone Joint Surg [Br]2007;89-B:925-7.

15. Jeng CL, Vora AM, Myerson MS. The medial approach to triple arthrodesis: indications and technique for management of rigid valgus deformities in high-risk patients. Foot Ankle Clin 2005;10:515-21.

16. Brilhault J. Single medial approach to modified double arthrodesis in rigid flatfoot with lateral deficient skin. Foot Ankle Int 2009;30:21-6.

17. Jeng CL, Tankson CJ, Myerson MS. The single medial approach to triple arthrodesis: a cadaver study. Foot Ankle Int 2006;27:1122-5.

18. Sangeorzan BJ, Mosca V, Hansen ST Jr. Effect of calcaneal lengthening on relationships among the hindfoot, midfoot, and forefoot. Foot Ankle 1993;14:136-41.

19. Toolan BC, Sangeorzan BJ, Hansen ST. Complex reconstruction for the treatment of dorsolateral peritalar subluxation of the foot. J Bone Joint Surg [Am] 1999;81-A:1545-60.

20. Sammarco VJ, Magur EG, Sammarco GJ, Bagwe MR. Arthrodesis of the subtalar and talonavicular joints for correction of symptomatic hindfoot malalignment. Foot Ankle Int 2006;27:661-6.

21. Fortin PT, Walling AK. Triple arthrodesis. Clin Orthop 1999;365:91-9.

22. O'Malley MJ, Deland JT, Lee K. Selective hindfoot arthrodesis of the treatment of adult aquired flatfoot deformity: an in-vitro study. Foot Ankle Int 1995;16:411-17.

23. Gelleman $\mathbf{H}$, Lenihan $\mathbf{M}$, Halikis $\mathbf{N}$, et al. Selective tarsal arthrodesis: an in vitro analysis of the effect on foot motion. Foot Ankle 1987;8:127-33.

24. Astion DJ, Deland JT, Otis JC, Keneally S. Motion of the hindfoot after simulated arthrodesis. J Bone Joint Surg [Am] 1997;79-A:241-6. 